\title{
Erratum to: Numerical modeling of flow due to consolidation of mine waste tailings
}

\author{
Mojtaba Nosrati $^{1} \cdot$ Emad Ghazavi Baghini $^{2} \cdot$ Mohammad Mohsen Toufigh $^{2}$
}

Published online: 2 June 2016

(C) Saudi Society for Geosciences 2016

Erratum to: Arab J Geosci

DOI 10.1007/s12517-016-2411-0

The original version this article, unfortunately, contained an error.

The first and third authors were unintentionally omitted. The complete list of authors and their affiliations are now present in this article.

The author list should be corrected as follows:

Mojtaba Nosrati · Emad Ghazavi Baghini · Mohammad Mohsen Toufigh

The online version of the original article can be found at http://dx.doi.org/ 10.1007/s12517-016-2411-0.

Emad Ghazavi Baghini ghazavi.emad@gmail.com

1 Middle East Water and Environment Inc., No.49, South Sheikh Bahaii St., MollaSadra Ave., Tehran, Iran

2 Department of Civil Engineering, Shahid Bahonar University of Kerman, 22 Bahman Blv., Kerman, Iran 